\title{
Exploiting the Capacity of Multichannel Multiradio Wireless Mesh Networks
}

\author{
Rongsheng Huang, Student Member, IEEE, Sunmyeng Kim, Chi Zhang, Student Member, IEEE, and \\ Yuguang Fang, Fellow, IEEE
}

\begin{abstract}
Current approaches for designing wireless mesh networks (WMNs) suffer from low-end-to-end-throughput performance. This disadvantage mainly comes from the traffic aggregation and multihop properties of WMNs. Although the throughput performance can be improved by utilizing multiple channels and multiple radios, the knowledge of how good the performance can be is still unavailable. Some previous works endeavored to heuristically improve the systems' performance, while others focused on the optimization algorithms for satisfying the clients' traffic demand. There is no work, until now, that can provide the knowledge of the system capacity. Although optimization algorithms have been proposed in previous papers, they require the exact traffic load input and make the "optimal" result easily outdated due to local topology and traffic changes. In this paper, we investigate the capability that WMNs can offer the mesh clients to deliver/receive data across the gateways, which is termed the portal capacity. Furthermore, we propose our solution to achieving the optimality in portal capacity through the centralized algorithm while retaining optimality via distributed tuning when local changes in either topology or traffic occur in the system. The concept of portal capacity provides us with very useful information about a WMN's capability, which can facilitate the optimization of the end-to-end throughput and fairness. In addition to this, this paper is the first one that takes the optimality and dynamic property of the system into consideration.
\end{abstract}

Index Terms-Channel assignment, multiple channels and multiple radios (MC-MR), resource allocation, wireless mesh network (WMN).

\section{INTRODUCTION}

W IRELESS MESH networks (WMNs) can be deployed to extend the coverage of last-mile access, which are seen as the extended version of wireless local area networks (WLANs) aiming at larger coverage areas and higher bit rates. Compared with its counterpart, i.e., cellular networks, WMNs

Manuscript received February 11, 2009; revised June 5, 2009. First published June 30, 2009; current version published November 11, 2009. This work was supported in part by the National Science Foundation under Grant CNS0721744. The work of Y. Fang was supported in part by the 111 Project under Grant B08038. The work of S. Kim was supported by Research Fund, Kumoh National Institute of Technology. The review of this paper was coordinated by Dr. H. Jiang.

R. Huang and C. Zhang are with the Department of Electrical and Computer Engineering, University of Florida, Gainesville, FL 32611 USA (e-mail: rshuang@ufl.edu).

S. Kim is with the School of Computer and Software Engineering, Kumoh National Institute of Technology, Gumi 730-701, Korea.

Y. Fang is with the Department of Electrical and Computer Engineering, University of Florida, Gainesville, FL 32611 USA, and also with the National Key Laboratory of Integrated Services Networks, Xidian University, Xi'an 710071, China (e-mail: fang @ece.ufl.edu).

Color versions of one or more of the figures in this paper are available online at http://ieeexplore.ieee.org.

Digital Object Identifier 10.1109/TVT.2009.2026421

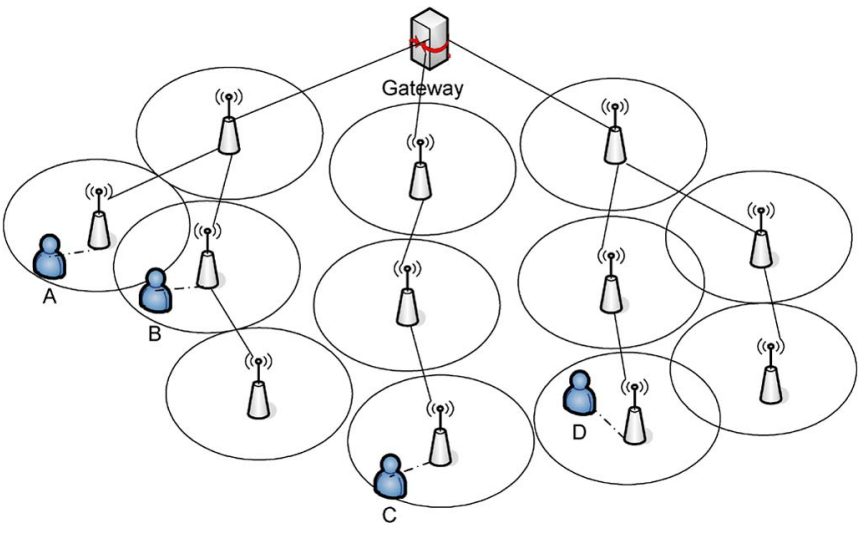

Fig. 1. Common structure of a WMN.

can provide mesh clients the ability to access the Internet with the advantages of low cost, ease of deployment and configuration, and flexibility of construction. For example, WMNs can provide Internet services to unscheduled conferences, military deployments, or emergency rescues.

Akyildiz et al. [1] have provided an excellent survey on WMNs. Commonly, a WMN consists of a limited number of gateways to access the wired networks, many mesh routers, each of which covers a certain area, and a larger number of mesh clients. Fig. 1 gives an example of a WMN structure with one gateway. Gateways function as portal devices, which provide access to the wired Internet. Mesh routers connect with gateways via wireless media through zero or more intermediate mesh routers. Mesh clients access the system through the local mesh routers. The local mesh routers help mesh clients to forward data packets to the gateways, with one or multiple intermediate hops.

Nowadays, two series of standards can be applied to WMNs. One is the contention-based IEEE 802.11 family, among which, the ongoing one, i.e., IEEE 802.11s, is aiming to better support the mesh structure. The other is the IEEE 802.16 family with the scheduling-based feature. It is widely known that the current IEEE 802.16 family does not support multihop networks well. IEEE 802.16j, which aims at multihop applications and is also known as mobile-multihop-relay-based WiMAX, is still under development.

Usually, mesh clients use WMNs mostly to access the Internet. Therefore, the ability for a WMN to provide mesh clients to deliver/receive data across the gateways is the focus of the design. Due to the limited number of gateways and the irregular distribution of mesh clients with multihop distance from the gateways, it is difficult to construct a WMN that 
can satisfy both coverage and throughput requirements. Researchers have been delving into the design issues. Jiang et al. [2] proposed a resource-management scheme based on codedivision multiple access for the wireless mesh backbone. To boost the throughput of WMNs, they design the scheme of resource allocation by using location and interference information from the receivers' point of view. Attempting to find out what attribute is responsible for the current low-throughput performance of WMNs, we generalize two major features for WMNs: multihop and traffic aggregation.

Due to the multihop feature of WMNs, a large part of resource is "wasted" in forwarding at the intermediate routers. This feature causes multiple channel contentions and flow discontinuity as well. The other major difficulty coming from the multihop feature is the frequency reuse or, looking from another angle, the concurrent transmission. When there is no frequency reuse, the system throughput is definitely low because so many links share a limited amount of frequency resource, a large part of which is used for forwarding. In multihop scenarios, reusing faraway frequency resource with proper planning is a basic and effective way to improve the throughput performance.

Because of the traffic aggregation toward a gateway, central links always have more traffic load. When these links do not acquire more resource than outside links, congestion is created, and bottlenecks are formed. Generally, when a large amount of undeliverable traffic is generated in some area, the congestion creates bottlenecks. Several other reasons can create bottlenecks in WMNs as well. Due to the different sensing ranges among different routers, hidden-/exposed-terminal problems and blocking problems haunt WMNs. The hidden-terminal problem can increase the collision of a link. The exposedterminal problem and the blocking problem can create disadvantaged links. These abnormalities are all possible reasons for bottleneck formation.

Fortunately, with the aid of multiple channels and multiple radios (MC-MR), the WMNs' performance can be improved with effective channel allocation [3]. IEEE 802.11b and IEEE 802.11 a specify 3 and 12 nonoverlapping channels, respectively. To this end, many previous works proposed architectures or algorithms with different techniques [3]-[9].

Unfortunately, up to now, no previous paper has given us the knowledge of the capability that WMNs provide the mesh clients to deliver/receive data across the portals. It will be very useful to know the maximum traffic a WMN can support to deliver across the gateways at the construction phase without the presence of mesh clients.

In this paper, we investigate the capacity that WMNs provide mesh clients to deliver/receive data across the gateways, which we term as the "portal capacity." It will be shown later that the portal capacity varies with different fairness constraints. We assume that the traffic distribution is known so that, statistically, each mesh router contributes a certain amount of traffic to the gateways. Under these assumptions, the objective function of the optimal portal capacity is formulated without the requirement of the knowledge of the real-time traffic demand. Furthermore, we propose our solution for achieving the optimality in portal capacity through the centralized algorithm while retaining the optimality via a distributed mechanism.
The contributions of this paper are listed as follows.

1) It proposes the concept of portal capacity, which characterizes the wireless access capability of WMNs provided to mesh clients.

2) It formulates a target problem for maximizing the portal capacity with the fairness constraint and reduces it to a much simpler and more soluble one via frequency reuse.

3) It gives two parts of a solution in which the centralized part focuses on the realization of the optimum portal capacity, and the distributed part renders flexibility and robustness to the system.

The rest of this paper is organized as follows. Section II discusses the related works. Section III formulates the target problem. Section IV describes the proposed solution. Simulation and evaluation are provided in Section V. Conclusions are given in the final section.

\section{RELATED WORKS}

Some previous works proposed heuristic approaches utilizing MC-MR to increase the throughput performance [7]-[10]. In [8], an architecture called Hyacinth is proposed for the MC-MR WMNs. Each mesh router carries out load-balancing routing and load-aware channel assignment in a distributed fashion. In [7], interference mitigation is introduced in the design. The multiradio conflict graph and a breadth-first searching algorithm are used for the interference-aware channel assignment. In [10], the channel assignment is jointly designed with multipath routing and scheduling. In [9], a superimposed code is used in channel assignment because of its s-disjunct property. By assigning a superimposed code to each mesh router in advance, the communication channel can be determined by the manipulation of two superimposed codes. However, this method has a requirement for a relatively large number of channels to avoid the cochannel interference. Most of these heuristic works are distributed schemes, which can quickly adapt to the traffic variation and link failures; thus, robustness can be achieved, although they lack consideration of the optimality of the throughput performance.

Some works instead apply optimization techniques to achieve the optimality of the system [4]-[6], in a centralized manner. Both [5] and [6] use a scale factor $\lambda$ to scale the flow rate under the constraints in WMNs, while they use different interference models and different algorithms to solve the optimization problems. In [4], the target problem is to directly maximize the summation of the utility function of the rate for each flow. These works are mostly too complicated in terms of the algorithm because of the need to decompose the NPhard optimization problem and because of the lack of robustness to support a real system. Meanwhile, the aforementioned algorithms do not give us information about the capability that a WMN can provide its clients to deliver packets across the gateways.

In addition, to achieve and retain the optimality in these centralized works, WMNs are always assumed to have a stationary mesh topology and static traffic demand when calculating the optimal value. Although gateways and mesh routers are mostly stationary, due to inevitable wireless link failures, occasional 
node failures, and node maintenance, the topology cannot be considered immutable. Moreover, although mesh routers usually have a more constant traffic load than mesh clients by aggregating the mesh clients' traffic, the traffic loads of mesh routers can still greatly vary from time to time due to a variety of group events, just like city traffic. Consequently, with the aforementioned algorithms, any of these local minor changes in the network can cause loss of fairness and optimality, which requires further global channel assignment and resource allocation. Therefore, the variation in topology and traffic load cannot be ignored in WMNs' design. Although the relatively stable and stationary information of the topology and traffic can be the input for calculating the optimal performance, a dynamic mechanism is also required to address the robustness issue.

\section{PROBLEM Formulation}

\section{A. Portal Capacity}

Unlike other ad hoc networks, a WMN is usually deployed by placing a number of mesh routers around one or several gateways so that mesh clients are able to deliver/receive data across this infrastructure and access the Internet via portal devices, i.e., gateways. (In this paper, we first investigate the scenario with one gateway.) Obviously, the throughput that the gateways carry is the aggregate end-to-end throughput of the system. Previous works investigated the aggregate end-toend throughput performance with the knowledge of the realtime traffic demand from mesh clients. However, the wireless ability that a WMN provides its clients to deliver/receive data across the gateway/gateways has never been touched. Hereafter, we refer to this wireless ability as the portal capacity. It is obvious that each mesh router in a WMN takes its capacity to/from the gateway as its individual portal capacity for its local mesh clients.

Similar to access points in WLANs, the maximum achievable throughput of gateways in WMNs defines the capacity of the whole system. The calculation of a WLAN's capacity is simply the capacity of the operating channel due to its one-hop nature. In multihop WMNs, the system capacity is not a constant as it is in WLANs because the spectrum is shared by multihop links. Different spectrum resource sharing among multihop links will cause different portal capacities. According to the traffic aggregation property, the system capacity can be learned via the summation of the capacity of the interfering last-hop links connected to the gateways. Without considering fairness, a WMN's portal capacity reaches the maximum value when the last-hop links take all the channel resource, while all other mesh clients and mesh routers are starved. Therefore, the portal capacity is meaningful only when the fairness constraint is considered.

If fairness can only be achieved when each link's traffic demand is satisfied with the predefined proportion, as in [6], the retainment of this fairness will be too difficult because each time the traffic of any mesh client changes, fairness will change, and the traffic should be rescheduled. Due to this consideration, we consider fairness in another way. We assume that the traffic distribution follows a fixed probability density function. The resource allocation can be based on the knowledge of this distribution so that the optimum portal capacity can be

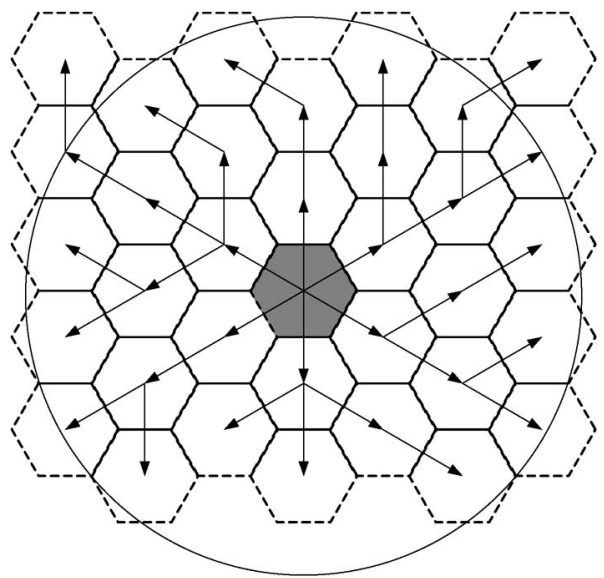

Fig. 2. Example of the link distribution in WMNs and the proposed frequency reuse pattern.

achieved. In addition, a dynamic mechanism is necessary to adjust this resource allocation to efficiently use up the spectrum in real time. In this paper, we assume that the traffic follows a uniform distribution and that the coverage of each mesh router is identical. Consequently, each mesh router is seen to be equivalent with respect to its traffic contribution to the gateway (with the forwarded traffic from other mesh routers excluded). Obviously, this problem seems to be much easier to solve without considering the real-time traffic demand of mesh clients. The optimal portal capacity under this fairness constraint is then determined only by the infrastructure consisting of the gateway, the mesh routers, and the links among them, which are relatively stationary. The knowledge of the portal capacity can thereupon be obtained without the presence of mesh clients at the construction phase and provide the engineers with important information beforehand.

Although this model ignores the diversity of traffic demand among the clients, it can give us useful knowledge of the system's performance and guide the design of the system. Such information cannot be obtained if the real-time traffic demand is considered for fairness. Furthermore, we can statistically incorporate the diversity of traffic demand by giving different traffic weights to different mesh routers according to their geographic characteristics. For example, in busy areas such as shopping malls or important areas such as hospitals, the mesh routers can have bigger traffic weights than others.

\section{B. Problem Formulation}

Apparently, the optimum portal capacity of the system and its realization are our focus in this paper. We denote the set of final-hop links to the gateway(s) as $\mathbb{L}_{1}$, the set of mesh routers as $\mathbb{M}$, and the predefined traffic demand weight for mesh router $k$ as $w_{k}$. The portal capacity via final link $i$ is denoted as $P_{i}$. The final links are those ones converged to the shaded area (the gateway) in Fig. 2. The aggregate portal capacity without consideration of fairness can be expressed as $\sum_{i \in \mathbb{L}_{1}} P_{i}$. Mesh router $j$ 's proportion of the portal capacity via final link $i$ is denoted as $u_{i j}$

$$
\max \sum_{i \in \mathbb{L}_{1}} P_{i} \sum_{j \in \mathbb{M}} u_{i j}
$$


subject to

1) the link capacity constraint;

2) the channel resource and radio resource constraint;

3) the interference (concurrent transmission) constraint;

4) the fairness constraint: $\sum_{i \in \mathbb{L}_{1}} u_{i j} P_{i}=\left(w_{j} / \sum_{k \in \mathbb{M}} w_{k}\right)$ $\sum_{i \in \mathbb{L}_{1}} P_{i}$.

This formulated problem searches in all the possible resource allocation and channel assignment for the maximum portal capacity with the fairness constraint. The fairness constraint requires each mesh router to share a proportional amount of the portal capacity corresponding to its traffic demand weight $w_{k}$. The objective function embeds $u_{i j}$ into each $P_{i}$ to add the fairness constraint. This is still an NP-hard problem, even after it removes the requirement on traffic demand. However, we can further simplify this problem.

\section{Problem Simplification}

We know that frequency reuse is critical to the system's performance because it can actually bring in more resource for allocation, particularly when the system covers multihop distance. However, in the backbone of WMNs, a good frequency reuse pattern is difficult to find.

Usually, in WMNs, the gateways are taken as the centers, and mesh routers surround the gateways with different distances, which are most of the time counted in the number of hops. Due to the traffic aggregation, the closer to the portal the links are, the heavier the traffic that they carry becomes. It is easy to observe that if a virtual circle is drawn in a WMN and the circle is large enough, links outside the circle can totally reuse the resource of the inside links, as shown in Fig. 2. The first reason for the feasibility of this frequency reuse is that outside links have less traffic load in total because all traffic needs to be aggregated in the gateway. The second reason is that the distance between frequency reuse links can be guaranteed by the proper frequency reuse distance $D$. Finally, because of the larger area and less traffic, this reuse can be repeated when the distance to the gateway is larger. Therefore, we can reduce the original problem to a problem with a smaller area of interest. We term this circle with radius $D$ as circle $D$. We call the mesh routers outside circle $D$ the outside mesh routers.

The criterion to discover the minimum circle $D$ is that all the links outside the circle can reuse the frequency resource used inside the circle. Usually, the sensing range of a node is about twice as long as its transmission range (simulator ns-2 adopts the typical value 2.2). Therefore, in this paper, we set distance $D$ equal to three-hop coverage. It is clear that the links outside circle $D$ are always able to reuse some frequency resource used by some inside links with proper assignment. This frequency reuse separation remains valid no matter what the real topology is for the aforementioned reasons.

Therefore, the former transformed problem can be reduced to a problem with fewer constraints

$$
\max \sum_{I \in \mathbb{L}_{1}} P_{i} \sum_{j \in \mathbb{M}} u_{i j}
$$

subject to

1) the link capacity constraint within circle D;

2) the channel and radio resource constraint within circle D;

3) the interference constraint within circle $D$;

4) the fairness constraints: $\sum_{i \in \mathbb{L}_{1}} u_{i j} P_{i}=\left(w_{j} / \sum_{k \in \mathbb{M}} w_{k}\right)$ $\sum_{i \in \mathbb{L}_{1}} P_{i}$.

Note that we do not loosen the fairness constraints since each link within circle $D$ is required to carry the traffic from downstream routers as well. To unify the concerned area, the traffic demand weights of outside mesh routers are to be merged to the inside mesh routers. We assume that traffic from one outside mesh router is bound to a certain inside mesh router on the boundary of circle $D$. Although this assumption means that the optimality is based on a certain fixed routing, it does not change the optimality when the routing is changed. This can easily be demonstrated as follows. We first assume that each router has enough radios that can support as many resources as it has been allocated. Each link is allocated resources according to its weight. As long as the total weight is not changed, e.g., each mesh router has a fixed hop distance to the gateway, then each mesh router can always obtain the same amount of portal capacity. Otherwise, the optimality might be changed, while the system performance would not have a great impact in that this would not bring a significant weight change. In reality, the number of radios cannot be as large as required. An improper routing does make some links overloaded, which would affect the portal capacity. However, this difference rarely makes the nonoptimality happen because the limited number of channels makes it impossible for a link to use more than one radio. Therefore, a small variation in the links' weights does not significantly change the system's optimal performance. In our solution, we use the simple tree structure routing. Although this routing suffers from lack of robustness, a dynamic adjustment mechanism can make up for this defect.

Based on this tree structure, the last-hop links are aware of their load, i.e., the total traffic they are carrying. Therefore, the proportions of all the $P_{i}$ can be acquired. Hence, $\mu_{i j}$ can be known if multipath routing within circle $D$ is ignored. With the given tree structure and without considering frequency reuse, the simplified problem becomes a linear programming (LP) problem within circle $D$ since the objective function and constraints are all linear. If the accumulated weights of the finalhop links change, a new global resource allocation and channel assignment is needed to regain the fairness.

\section{Proposed Solution}

Unlike previous papers related to the optimality, our solution is based on the standard of the IEEE 802.11 family because the frame structures in IEEE 802.16 standards cannot support multihop very well, even though the inherent scheduling mechanism can greatly reduce the contention overhead in IEEE 802.11. Note that we assume that the links between mesh routers and their local mesh clients use a different set of frequency bands so that they can be ignored in this paper and that the network planning about this part can directly refer to the cellular systems. 
From the problem formulation, we have found a new way to achieve WMNs' optimality. Assuming that each wireless link within circle $D$ has the same wireless condition and that each wireless router has at least two radios (with one for uplink and the other for the downlink) and ignoring the frequency reuse, the aforementioned LP problem can easily be solved by allocating the available bandwidth of given multiple channels to links among mesh routers according to their weights. Therefore, the key to the optimal portal capacity is the resource allocation and channel assignment for the links within circle $D$.

For a practical system scheme, allocating channel resource to links within circle $D$ to achieve an optimal aggregate portal capacity is not enough. First, each outside mesh router should have a way to acquire a certain amount of resource that supports the optimal portal capacity. Second, as aforementioned, outside mesh routers can totally reuse the spectrum resource within circle $D$. A scheme is required to realize this frequency reuse that prevent the links within circle $D$ from being interfered by outside links.

Finally, the achieved optimality requires the condition that each mesh router generates the preplanned traffic load. Realtime traffic varies from the expected value from time to time; thus, the calculated resource allocation may not result in the optimal portal capacity. With a distributed and dynamic mechanism to adjust the allocated resource, when traffic varies, the optimal portal capacity can still be maintained. Robustness is also gained through the distributed and dynamic scheme.

The overall proposed solution consists of a centralized part and a distributed part, which are discussed next, respectively.

\section{A. Part I: Centralized Part}

1) Overview: The optimal portal capacity is mostly determined by the resource allocation and channel assignment for the links within circle $D$. The resource allocation here only means the allocation of bandwidth resource of given channels to the available radios of the mesh routers. Although the difference in allocated resource may be limited by the number of available radios of the considered mesh routers, this probability is rather low because, due to the limited number of channels, one link can rarely occupy more than one channel.

We propose a centralized scheme to carry out the task of resource allocation and channel assignment.

As aforementioned, with the assumption of uniform traffic distribution and identical coverage area among mesh routers, each mesh router contributes the same amount of traffic to the gateway. Therefore, the weight of each link can be defined as the number of downstream mesh routers. Without the consideration of frequency reuse, the resource allocation is straightforward with the knowledge of the total resource and the links' weights. A weighted allocation is enough for this task. Afterward, we need to face the challenges from frequency reuse, exact total resource, and the deviation of resource allocation.

Some notations are introduced beforehand. The channel capacity is first assumed as a constant $B$, and there are $K$ available channels. We denote the total weight of each tier as $\omega_{\mathrm{I}}, \omega_{\mathrm{II}}$, and $\omega_{\text {III }}$, respectively. The total weight is $\omega_{\text {total }}$. The numbers of each tier's mesh routers are denoted as $M_{\mathrm{I}}, M_{\mathrm{II}}$, and $M_{\mathrm{III}}$, respectively. The number of all mesh routers is denoted as $M$.

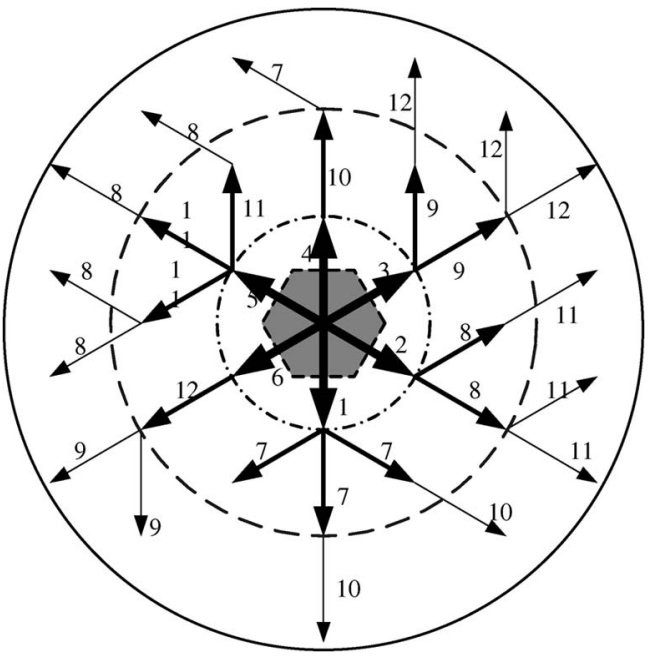

Fig. 3. Ideal case when frequency reuse is perfect.

Conservatively, when there is no frequency reuse within circle $D$, a lower bound of the portal capacity can be achieved by dividing the whole spectrum by the whole weights of the links. The lower bound is $K \cdot B \cdot M /\left(M+\left(M-M_{r m I}\right)+\right.$ $\left.\left(M-M_{\mathrm{I}}-M_{\mathrm{II}}\right)\right) \approx K \cdot B / 3$ when $M_{\mathrm{I}}, M_{\mathrm{II}} \ll M$.

An upper bound is achieved when the third-hop links can all reuse the frequency allocated to the preceding links, as shown in Fig. 3. To achieve this upper bound, the network topology and the links' weights should roughly be symmetric to the gateway. The most ideal aggregate portal capacity is $K \cdot B \cdot M /(M+$ $\left.\left(M-M_{\mathrm{I}}\right)\right) \approx K \cdot B / 2$ when $M_{\mathrm{I}} \ll M$.

Therefore, the achievable value range for problem (2) is known to vary from $K \cdot B / 3$ to $K \cdot B / 2$. However, if the scheme is not well designed, the real system's aggregate portal capacity can be far lower than these values. From this section onward, we describe the scheme of exploiting the portal capacity of WMNs.

For an IEEE 802.11 channel, the maximal throughput $B$ that can be achieved is not a constant. It depends on the number of contending nodes, i.e., the collision probability. If the channel's maximal throughput is not the same for different links, the weighted resource allocation is not accurate, which may lead to the underutilization of the portal capacity or a nonoptimal portal capacity. The real channel capacity with different numbers of contending nodes should be figured out, and the capacity difference needs to be considered in the resource allocation. A revised weighted allocation is used for this purpose.

We first present the algorithm for the centralized resource allocation and channel assignment with the consideration of frequency reuse.

2) Centralized Resource Allocation and Channel Assignment: It can easily be observed that the difference in the upper bound and the lower bound of the aggregate portal capacity comes from the difference in frequency reuse. Obtaining the frequency reuse as much as possible leads to the optimal aggregate portal capacity.

The most common way to allocate resource is using a weighted allocation. $R_{i}=\omega_{i}\left(R_{\text {total }} / \omega_{\text {total }}\right)$, where $R_{i}$ stands for the allocated resource to link $i$, and $R_{\text {total }}$ stands for the 
total resource. When one link can reuse another link's resource, its weight $\omega_{l^{\prime}}$ (which is assumed to be not greater than the reused one's weight $\omega_{l}$ ) is not counted again; thus, the share $R_{\text {total }} / \omega_{\text {total }}$ will be increased due to a smaller total weight.

Based on the rule that frequency cannot be reused by two-hop neighbors [4], we can derive another two rules: the frequency of first-tier links cannot be reused by third-tier links, nor can the second tier be reused by the second tier. Therefore, it is clear that the frequency reuse for the links within three hops is only possible between faraway second- and third-tier links or between faraway third-tier links. From an engineering point of view, it is not difficult to figure out all the reused link pairs within circle $D$ during network construction. This information can be configured into gateways for the centralized resource allocation.

After allocating the proportional resource to each link, the gateway is required to assign the channels to the radios of each mesh router according to the allocated resource to each link. Because the number of channels is an integer, the channel assignment can make the resource allocation deviate from the target proportion due to rounding operations. A fittest-allocation strategy is used to mitigate this problem. The frequency-reusing links do not need to be assigned channel resource because of a previous assignment for the frequency-reused links.

Due to the limited number of channels, most links in WMNs have to share a channel with other neighboring links. This fact imposes extra difficulty on the frequency reuse. Under a contention-based protocol, if the frequency reused links share the channel with other links, the frequency reuse is not always feasible. An example is that channel assignment makes a first-tier link share a channel with its downstream third-tier link. In this case, its downstream fourth-tier links cannot reuse the frequency resource of this first-tier link because it will bring undesired interference between the third-tier link and the fourth-tier links.

When the frequency reuse cannot be fulfilled under a certain channel assignment, the resource allocation is required to recalculated due to the change in total weight $\omega_{\text {total }}$. Therefore, the resource allocation needs to be jointly considered with channel assignment due to the uncertainty of frequency reuse. Our strategy is to compare the gain from frequency reuse and the cost to exclude other links from the frequency-reused channel.

Resource allocation is first done with the knowledge of total weight $\omega_{\text {total }}$ and total resource $R_{\text {total }}$. During the channel assignment, when a frequency-reused link is met, we attempt to exclude all the links that cannot be frequency reused from the current channel and quantify this resource as virtual weight $\omega_{\text {virtual }}$. The gain of this frequency reuse is the saved resource for these reused links, which are quantified by their weights $\omega_{l^{\prime}}$. If the cost is less than the gain, we add virtual weight $\omega_{\text {virtual }}$ to and subtract the gain $\omega_{l^{\prime}}$ from $\omega_{\text {total }}$ and redo the resource allocation. Otherwise, the frequency reuse for these links is abandoned. This procedure is repeated until all the links have been assigned channels. Note that if more than one link can reuse the same frequency reuse link set, the cost and gain comparison should consider all of them. Table I shows the pseudocode of the centralized resource allocation and channel assignment with the consideration of frequency reuse.
TABLE I

Algorithm of Centralized Resource Allocation AND CHANNEL ASSIGNMENT

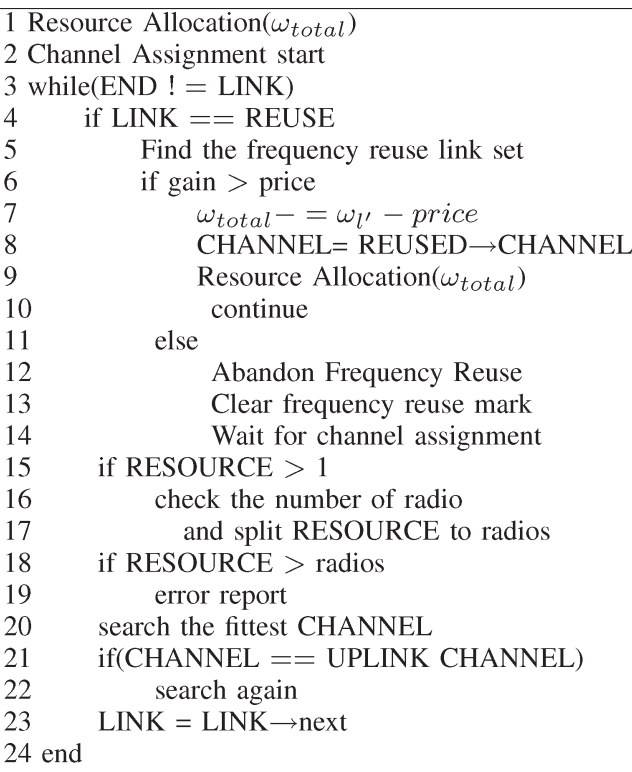

As shown in line 21, an important rule for the assignment is that the channel frequency for the uplink and downlink of each mesh router should be different. The constraint of the radio resource is also considered in line 15 . When there is more than one radio for downlink or uplink, the strategy is to evenly spread the allocated resource to each radio. This strategy considers the prospective dynamical adjustment so that, when the unused resource can be reallocated, an extra channel assignment is not necessary. The channel assignment should be continuous, which means that the same channel is preferred to be allocated to a close neighborhood. The purpose of this strategy is to avoid the hidden-terminal problem, as well as to enhance the frequency reuse probability.

3) Actual Total Resource for Allocation: The portal capacity being discussed does not mean the nominal channel bandwidth. This value is defined as the achievable throughput across the gateways. It is used by the parent nodes to indicate to the child nodes their feasible traffic rate. Each child node should follow this quota; otherwise, the parent cannot guarantee the delivery, and the optimal portal capacity cannot be guaranteed either. The allocated resource for each link is equal to the portal capacity that the child node inherits from the parent node, which will be shared by all the child node's downstream mesh routers.

Due to the traffic aggregation property of WMNs, the central links' weights are always greater than those of the outside links. Therefore, when weighted resource allocation is used, the central links usually get more resources than outside links. Therefore, in contention-based MC-MR WMNs, the number of sharing links closer to the gateways is much different from the one farther away from the gateways. For a contention-based protocol, the channel's capacity depends very much on the contention probability. When the number of links sharing the same channel is different, the actual capacity of this channel is different. According to the result of [11], this difference can be up to $10 \% \sim 20 \%$. If we use the nominal channel capacity as the 
resource for allocation, the central links' allocated resource will greatly exceed their target proportion. Eventually, the optimality will be lost. To exploit the realistic optimal portal capacity, the first task is to find out the actual resource for allocation.

The saturation throughput of IEEE 802.11 channels has been given out in [12]. However, this throughput cannot be used to indicate the child nodes' traffic rate. The saturation throughput means the real delivery rate in IEEE 802.11 systems when each node has packets to send all the time. The traffic rate (arrival rate) of the senders is usually much larger than the delivery rate in this case. We do not want to use an arrival rate that is too big to achieve the target delivery rate under saturation because an overstocked system is not preferred. A mapping from the arrival rate to the delivery rate under nonsaturation is required for our purpose.

For analysis purposes, we assume that the arrival of packets for one mesh router is modeled as a Poisson process. The packet delivery of a station can be modeled as a queue system, and the medium-access-control (MAC) service time is the service time of this $\mathrm{M} / \mathrm{G} / 1 / \mathrm{K}$ system when the system is nonsaturated. When the arrival rate is greater than the service rate, this system is overstocked, i.e., nonergodic. We can find the biggest nonoverstocked arrival rate to get the knowledge of the achievable delivery rate, which is the value used to indicate the child nodes. The following is the procedure to get such actual channel capacity.

We denote the number of contenders in one channel as $n_{c}$, and each contender is assumed to have the same packet arrival rate $\lambda_{c}$. According to the result of [11], the MAC service rate $\mu$ can be acquired as the inverse of the average service time $1 / E\left(T_{s}\right)$.

To make the queue system nonoverstocked, the arrival rate should be less than the service rate

$$
\lambda_{c}<\mu=\frac{1}{E\left(T_{s}\right)} .
$$

The maximum arrival rate can be achieved when the following equation holds: $\lambda_{c}=\left(1 / E\left(T_{s}\right)\right)$.

The maximum acceptable traffic load of this channel can be achieved through this process. Thus, the maximum throughput is

$$
S=n_{c} \lambda_{c}\left(1-p_{B}\right)\left(1-p_{c}^{\alpha+1}\right)
$$

where $p_{B}$ is the packets' dropping probability due to a finite queue length, $p_{c}$ is the packet discard probability in one transmission, and $\alpha+1$ is the retransmission time. The derivation can also be found in [11].

However, when each contender has a different arrival rate, these values may change.

We rewrite the relationship between $p_{c}$ and conditional transmission probability $\tau$ as

$$
p_{c}(j)=1-\prod_{i \neq j}^{n_{c}}\left(1-\left(1-p_{0}(i)\right) \tau_{i}\right)
$$

where $p_{0}(i)$ is the probability that wireless station $i$ has no packet to transmit, which can be derived from $\lambda_{c}(i)$. Mean- while, from the Markov chain model, the conditional transmission probability can be derived as

$$
\tau_{i}= \begin{cases}\frac{2\left(1-p_{c}(i)^{\alpha+1}\right)}{1-p_{c}(i)^{\alpha+1}+\left(1-p_{c}(i)\right) W \sum_{k=0}^{\alpha}\left(2 p_{c}(i)\right)^{k}}, & \alpha \leq m \\ \frac{2\left(1-p_{c}(i)^{\alpha+1}\right)}{1-p_{c}(i)^{\alpha+1}+p_{c}(i) W \sum_{k=0}^{m-1}\left(2 p_{c}(i)\right)^{k}+\left(1-2^{m} p_{c}(i)^{\alpha+1}\right)}, & \alpha>m\end{cases}
$$

where $m$ is the maximum number of the stages allowed in the back-off procedure.

From these two equations, $p_{c}(i)$ can be figured out with the input of $n_{c}$ and $\lambda_{c}(i)$.

When $\lambda_{c}(i)$ are provided, $p_{c}(i)$ can be figured out. The average MAC service time for each station can also be calculated. The total throughput is the summation of each station's throughput, which can be derived from $\lambda_{c}(i)$ and $p_{c}(i)$.

An extreme case of different arrival rates is that, among $L$ contenders, only one has intense traffic, and the others sparsely transmit. The maximal throughput in this case approximates the one in the case that $n_{c}$ is equal to 1 .

This time-varying channel capacity adds more difficulty to the centralized resource-allocation task. However, it is shown that the unequal traffic pattern leads the maximal throughput to approximating the value with fewer contenders. Therefore, the equal-traffic case is reasoned to have the most conservative channel capacity, and this value is used as the input in our resource allocation scheme.

4) Revision of Weighted Resource Allocation: With different numbers of contending nodes $n_{c}$, the maximum arrival rate can be acquired from the aforementioned process. We can use factor $\eta$ to indicate the actual channel capacity as $\eta B$. Since the number of contending nodes for same-tier links does not greatly vary, we can assume the $\eta$ for same-tier links to be the same. The factors for different tiers can be denoted as $\eta_{\mathrm{I}}, \eta_{\mathrm{II}}$, and $\eta_{\mathrm{III}}$ for tiers 1,2 , and 3 , respectively.

The derivation of each tier's average number of contending nodes $\left(C_{\mathrm{I}}, C_{\mathrm{II}}\right.$, and $\left.C_{\mathrm{III}}\right)$ can easily be obtained as follows:

$$
\begin{aligned}
C_{i} & =\frac{M_{i}+1}{K \frac{\omega_{i}}{\omega_{\text {total }}}} \\
C_{\mathrm{II}} & =\frac{M_{\mathrm{II}}+M_{i}}{K \frac{\omega_{\mathrm{II}}}{\omega_{\text {total }}}} \\
C_{\mathrm{III}} & =\frac{M_{\mathrm{III}}+M_{\mathrm{II}}}{K \frac{\omega_{\text {III }}}{\omega_{\text {total }}}} .
\end{aligned}
$$

Through the weighted allocation process, the allocated resource for three tiers is $\eta_{\mathrm{I}} \omega_{\mathrm{I}} /\left(\eta_{\mathrm{I}} \omega_{\mathrm{I}}+\eta_{\mathrm{II}} \omega_{\mathrm{II}}+\eta_{\mathrm{III}} \omega_{\mathrm{III}}\right)$, $\eta_{\mathrm{II}} \omega_{\mathrm{II}} /\left(\eta_{\mathrm{I}} \omega_{\mathrm{I}}+\eta_{\mathrm{II}} \omega_{\mathrm{II}}+\eta_{\mathrm{III}} \omega_{\mathrm{III}}\right)$, and $\eta_{\mathrm{III}} \omega_{\mathrm{III}} /\left(\eta_{\mathrm{I}} \omega_{\mathrm{I}}+\eta_{\mathrm{II}} \omega_{\mathrm{II}}+\right.$ $\left.\eta_{\mathrm{III}} \omega_{\mathrm{III}}\right)$, respectively. The difference among $\eta_{\mathrm{I}}, \eta_{\mathrm{II}}$, and $\eta_{\mathrm{III}}$ means that the allocation greatly deviates from the target, implying that the actual resource allocated to three tiers should have the proportion of $\omega_{\mathrm{I}}, \omega_{\mathrm{II}}$, and $\omega_{\mathrm{III}}$, respectively.

The solution to correct this deviation is to use $1 / \eta_{\mathrm{I}}, 1 / \eta_{\mathrm{II}}$, and $1 / \eta_{\text {III }}$ to modify the weights of links for different tiers. For example, $\omega_{\mathrm{I}}^{\prime}=\omega_{\mathrm{I}} / \eta_{\mathrm{I}}$. Given that link $i$ is in tier 1, the resource allocated to it is

$$
R_{i}=K B \eta_{\mathrm{I}} \frac{\omega_{i}^{\prime}}{\omega_{\text {total }}^{\prime}}=K B \frac{\omega_{i}}{\omega_{\mathrm{I}} / \eta_{\mathrm{I}}+\omega_{\mathrm{II}} / \eta_{\mathrm{II}}+\omega_{\mathrm{III}} / \eta_{\mathrm{III}}} .
$$


The total allocated resource is then

$$
R_{\text {total }}=K B \frac{\omega_{\text {total }}}{\omega_{\mathrm{I}} / \eta_{\mathrm{I}}+\omega_{\mathrm{II}} / \eta_{\mathrm{II}}+\omega_{\mathrm{III}} / \eta_{\mathrm{III}}} .
$$

Through this correction, the target proportion of allocation can be attained.

\section{B. Part II: Distributed Part}

1) Overview: The centralized part of our solution provides a straightforward way to optimize the portal capacity of an MCMR WMN. To fully fulfill this optimum value, a distributed mechanism is needed as well. This includes not only the distributed mechanism for outside mesh routers to handle the channel assignment and the portal capacity fulfillment but a dynamic adjustment mechanism for each router to dynamically and fully utilize the portal capacity as well.

There are a couple of reasons why the distributed mechanism is preferred in addition to the centralized part. The first reason is that a distributed scheme can fit the dynamic environment better than a whole centralized one. For a centralized scheme, whenever this network is changed in either topology or traffic dynamics, an updating of the channel assignment is needed. A distributed scheme can update the channel assignment in response to local minor changes. The second reason is the difficulty of frequency reuse decision in multihop scenarios. For the centralized approach, to decide the whole frequency reuse, it requires the center gateway to know all the knowledge of the topology, i.e., the connectivity graph, which needs significant overhead to collect and maintain. A distributed scheme does not need this dependence and can cope with the frequency reuse according to the local knowledge and real-time information. The task of the outside mesh router reusing the frequency resource of the links within circle $D$ is also included in the proposed distributed scheme.

We have already demonstrated that when the central links have appropriately been allocated channel resources, the outside tiers should have enough channel resources to realize their share of the portal capacity. Given a mesh router's portal capacity, the task of its distributed channel assignment is to allocate to its children their shares of the portal capacity and assign them channels. The distributed algorithm can bring the network the adaptivity to possible minor local changes in traffic and/or topology, which should not cause an update across the whole network. The difficulties come from the acquirement of the status information of all the channels and the coordination of the channel assignment for neighboring mesh routers.

In addition, a dynamic mechanism is required to adjust the channel resource among different links after each of the mesh routers obtains its share of the portal capacity and the assigned channels. Moreover, this adjustment can help the mesh routers recover from the link failures and link disadvantages.

2) Distributed Channel Assignment: Even with all the links within circle $D$ acquiring their channel resource and fulfilling their portal capacity, the network still needs to address the channel allocation for outside mesh routers to gain their shares of the portal capacity. We propose a distributed scheme for the outside mesh routers to accomplish their portal capacity.

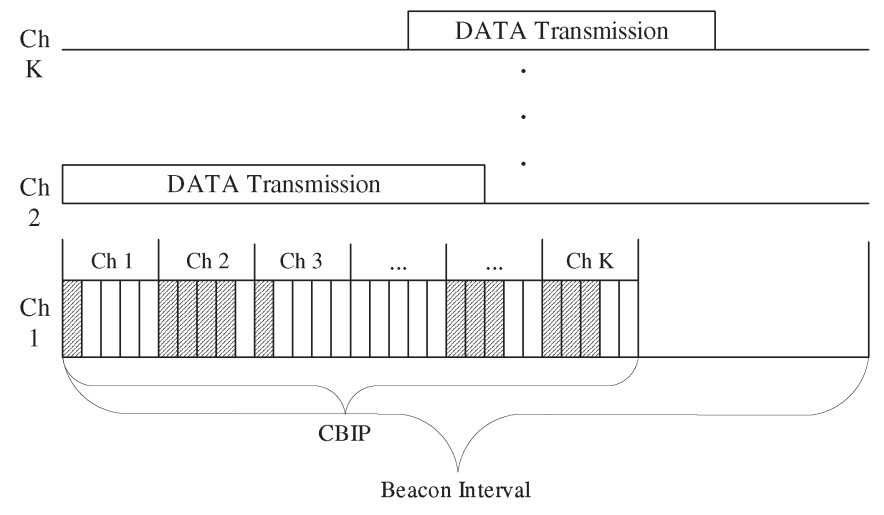

Fig. 4. Illustration of the CBIP.

Since the portal capacity of each mesh router is always passed down from its parent, the distributed channel assignment is determined to follow a top-down sequence. This means that the outside mesh routers can execute the distributed channel assignment only after the centralized resource allocation and channel assignment have been completed.

The centralized resource allocation is embedded in the procedure of the global channel assignment. Each mesh router waits for its parent to allocate the share of the portal capacity and assign the channel resource for it. A certain channel is defined as the starting channel. Before channel assignment, all mesh routers reside on the starting channel, waiting for the channel assignment from their parents or the gateway.

The major difficulty for a channel-assignment scheme with fewer radios than channels is the acquirement of the status information of all channels. Previous works use networkallocation-vector (NAV) knowledge collected from each of the channels to indicate the channel usage status for mobile ad hoc networks (MANETs) [13]. However, per-packet-based channel assignment does not fit WMNs because of the relatively stationary topology and relatively constant flow in the backbone links. Instead, the channel assignment in WMNs is almost static except that the current requirement cannot be satisfied. Due to the aforementioned reason, the NAV knowledge is not necessary, and a long-term indicator of the channel busyness level is preferred in the channel selection for WMN routers. We specify a certain duration at the beginning of every $T$ beacons on the starting channel as the channel busyness indicator phase (CBIP), as shown in Fig. 4. The CBIP is divided into $K$ parts, where $K$ is the number of available channels. Each mesh router sets busy tones on the corresponding parts to signal the busyness status of its operating channels, with the duration length as the busyness level. Each mesh router chooses the most idle and nonconflicting channel for the links to its children. The busy tone's length is always determined by the neighbor that senses the busiest channel status of this channel because the result of the addition of busy tones is the one with the longest duration. When a mesh router senses that some channel's busy tone is not set, it can tell that there is no neighbor using this channel.

The indicator of the channel busyness level is determined by the following formula:

$$
\delta=\left\lceil L \frac{T_{\text {busy }}}{T_{\text {total }}}\right\rceil
$$


where $L$ is the number of minislots used in the CBIP for one channel, $T_{\text {busy }}$ denotes the busy time duration of the given channel, and $T_{\text {total }}$ denotes the whole time duration. The granularity of this indicator is decided by $L$, which cannot be too large. However, in a WMN, the outside mesh routers greatly underutilize the channels because they reuse the frequency resource in a greatly larger area with significantly less traffic demand. Therefore, $L$ does not need to be large. For the mesh routers within circle $D$, although this indicator is not useful because every channel is fully allocated, this mechanism is also incorporated in the mesh routers within circle $D$ because of the neighborhood requirement from outside mesh routers and the requirement for a globally uniform protocol. Consequently, the centralized resource allocation is required to subtract this part of the resource.

The algorithm for the distributed channel assignment can be described as follows.

After a mesh router (three hops or farther) gets its portal capacity and channel assignment from its parent (or the gateway), it calculates the portal capacity that each of its children can get after it subtracts its own usage. It collects the busyness status of each channel via the CBIP and decides the channel assignment for its children afterward. For the case of more than one radio for downlink, the mesh router spreads the portal capacity to each available downlink radios for the convenience of prospective dynamic adjustment. After deciding the channel assignment or reassignment, both parent and child start to set the busy tone for this channel.

When the neighboring mesh routers carry out the channel assignment at the same time, it is possible that a certain channel is assigned by a lot of mesh routers and that other channels are spared. In our algorithm, each mesh router waits for all the higher tier routers to finish their channel assignment before it starts its own. Among the mesh routers with the same tier number, the mesh router carrying the largest weight starts first.

The overhead of this mechanism is the CBIP and one extra radio for the busy tone in the starting channel.

3) Dynamic Adjustment Mechanism: The dynamic adjustment mechanism handles two types of changes: infrastructureinvolved change and non-infrastructure-involved change.

If a mesh router's share of the portal capacity cannot be used up, its parent can let its siblings share this unused resource. Within circle $D$, the unused portal capacity can only be shared by links using the same channel because the channel resource is already fully allocated. Outside mesh routers have no such limit as long as they have vacant channel resource to fulfill the unused portal capacity.

This resource usage change is temporarily caused by traffic imbalance. This type of change has no impact on the relationship among mesh routers and is thereby defined as noninfrastructure-involved change. It also includes the case that a mesh router changes to another channel while keeping its share of the portal capacity. The triggering condition of this change can be a link disadvantage caused by the hidden-terminal problem or poor link quality due to frequency-selective fading and interference.

We can show that with only non-infrastructure-involved changes, the optimality of the current assignment is not changed, assuming that all the link statuses are identical. The proof is straightforward and is given as follows: As long as there is unused portal capacity due to some underutilized links, it can be used up by other overutilized links. Therefore, the optimum portal capacity will not lead to underutilization unless the total traffic demand is below the aggregate portal capacity.

A mesh router can monitor other mesh routers of higher tiers. It can change its parent when its portal capacity cannot be fulfilled via the current parent and there is an alternate parent who can provide a certain better share of the portal capacity. This change involves a change in the relationship of parent and child. It belongs to the infrastructure-involved change. The case of a new router joining the system and the case of a router detaching from its parent are also included in this type. The dynamic adjustment for infrastructure-involved change makes the system self-recoverable and might cause some unfairness in return. However, unfairness is the price for the robustness. Another global channel assignment can regain the fairness of the system.

For the sake of dynamic adjustment, a mesh router should broadcast its portal capacity and current usage ratio. A mesh router is also required to broadcast its tier number and weight and the number of downstream mesh routers.

4) Infrastructure Formation: When the system is being initialized, all the mesh routers carry out the association with the gateway on the starting channel. Each mesh router has a counter recording the number of downstream mesh routers and uses this counter as the initial weight to get the share of the portal capacity. In this initializing phase, each mesh router listens to the neighbors' broadcasting messages and finds the neighbor with the fewest hop counts to the gateway and the strongest signal strength as its parent. This way, the initial tree is formed. This tree structure can dynamically be adapted to link failures or load imbalance. The centralized resource allocation is always based on the WMN's current tree structure.

\section{Part III: Support of Multigateway}

In this paper, we only give a simple discussion of multigateway support with our solution.

Unlike other centralized approaches, our solution requires centralized resource allocation and channel assignment within circle $D$. Due to the smaller centralized area, supporting multiple gateways within one WMN becomes possible with our solution. When there are several gateways in the field, as long as the circle $D$ of each router is not overlapped, the links inside each circle can achieve their portal capacity from the gateways, and the links outside can acquire their portal capacity from any gateway in a distributed manner. To guarantee that the channel assignment of each circle do not interfere with each other, the separation distance between each circle should be at least two hops away. Although there are more issues to be considered when applying our solution to multigateway WMNs, our solution provides a good option that many previous works do not support at all.

With multiple gateways connected to the Internet, a WMN can provide a bigger portal capacity and a larger coverage area. 

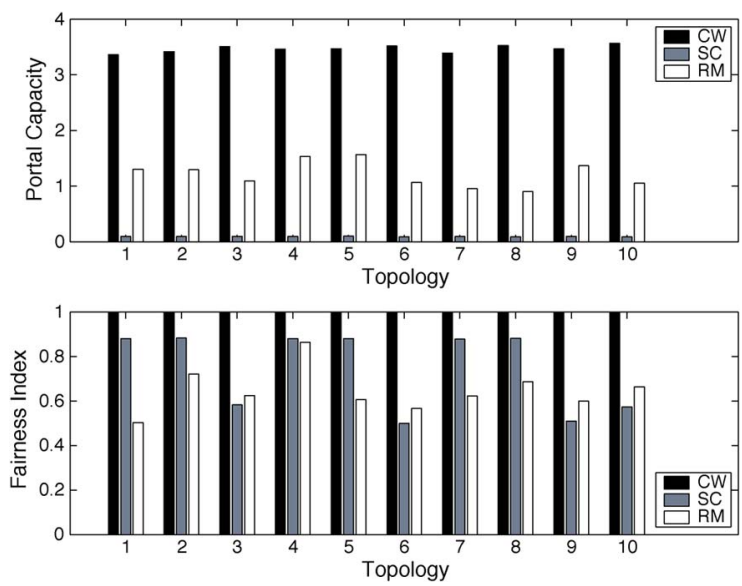

Fig. 5. Comparison of the portal capacity among three schemes. CW (our solution), SC (single channel), and RM (randomly choosing multichannel).

\section{Performance Evaluation}

Previous works have not proposed concepts similar to portal capacity; therefore, no knowledge is provided about how much channel resource can be transferred to the portal capacity in WMNs. In this paper, we propose a feasible solution leading to the optimum portal capacity. Since there is no previous result similar to the portal capacity, we use two simple schemes based on the current IEEE 802.11 standards for comparison. The first scheme for comparison is a distributed single-channel scheme (SC). With this scheme, each node in the system fairly contends for the total channel resource. The second scheme for comparison is a distributed multichannel scheme (RM), in which each node randomly picks a channel and contends for transmission. Using $\mathrm{C}$ program, we implement our proposed centralized algorithm of weighted resource allocation and channel assignment $(\mathrm{CW})$ to compare its the portal capacity performance with that of these two schemes, i.e., SC and RM. For the purpose of comparison, all three schemes are applied to topologies limited in circle $D$. We also assume that there is no frequency reuse in all three schemes and that there is no geographical disadvantage among all links. For both compared schemes, i.e., SC and RM, the portal capacity comes from the aggregation of the maximum throughput over the last-hop links. In both distributed schemes, since each node contends for the channel resource without considering the portal capacity share of its downstream nodes, the fairness has no guarantee. Thus, a fairness metric is included in the simulation as well.

This simulation sets $K$, which is the number of available channels, to 12 for our solution and the distributed multichannel scheme, i.e., $\eta_{\mathrm{I}}, \eta_{\mathrm{II}}$, and $\eta_{\mathrm{III}}$, to $1.0,0.8$, and 0.6 , respectively. The channel bandwidth $B$ is ignored in the simulation since it is a constant. Therefore, the allocated resource and portal capacity can be quantified by values ranging from 0 to 12 . We compare the value of the realized portal capacity among our centralized weighted allocation approach $(\mathrm{CW})$ and the compared schemes $\mathrm{SC}$ and RM.

Fig. 5 shows that in the SC case, only a small proportion of the total channel resource is transferred to the portal capacity or end-to-end throughput. The RM scheme can get better per-
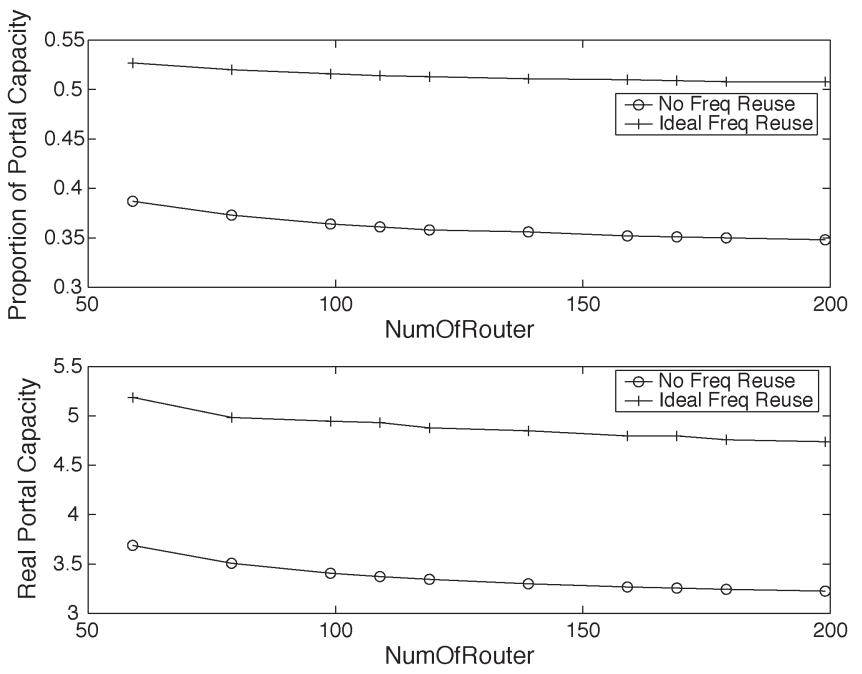

Fig. 6. Portal capacity with frequency reuse.

formance. With the channel-assignment overhead ignored, the gain of RM is roughly the same as $K$, which is still relatively low. Our solution (CW) can transfer about one third of the total resource to the portal capacity, which is roughly $200 \%$ more than that of RM.

As aforementioned, each mesh router is designed to be able to deliver the same amount traffic to the gateways generated by itself. If each link does not acquire the allocated resource according to the target proportion of the weight, the portal capacity cannot fairly be shared by all mesh routers. Therefore, we use $X_{i}$, which is the ratio between the allocated resource and the corresponding weight, to calculate the fairness index

$$
\zeta=\frac{\left(\sum X_{i}\right)^{2}}{n \cdot \sum\left(X_{i}\right)^{2}} .
$$

If the resource allocated to each link matches the corresponding weight, with the existence of admission control and message exchange between mesh routers, the fair share of the portal capacity can easily be reached. With the proper setting of the weight for each link, our solution achieves the best fairness, as shown in Fig. 5.

If the three schemes are applied to larger topologies, due to less planned resource allocation, SC and RM will perform much more poorly than our solution.

The second part of our simulation is focused on the frequency reuse issue. Frequency reuse can bring more actual channel resources and improved portal capacity. This part of the simulation shows the portal capacity improvement owed to frequency reuse in our solution. In our solution, centralized resource allocation only considers frequency reuse within circle $D$ because outside mesh routers can reuse the resource inside the circle. Therefore, frequency reuse inside circle $D$ determines the optimum frequency reuse of the whole system, which can easily be figured out through field measurements.

We test the portal capacity with ideal frequency reuse using our algorithm under the scenarios of different numbers of outside mesh routers. For comparison, the portal capacity without frequency reuse is also provided. 
Fig. 6 shows the portal capacity improvement using ideal frequency reuse. From the first part of Fig. 6, we can see a significant increase (roughly $40 \%$ ) of the proportion of the portal capacity, which means the ratio of the real portal capacity and the real allocated channel resource, not including the overhead and the unallocated channel resource. When the number of outside mesh routers changes, there is a slight decrease in both the portal capacity proportion and the real portal capacity. The reason is that, when the number of mesh routers increases, the weights of second- and third-tier links increase. Therefore, more channel resources will suffer from a smaller $\eta$.

\section{CONCLUSION}

This paper has attempted to investigate the capability that a WMN provides its mesh clients to deliver/receive packets across the gateways. This capability can be quantified by the defined portal capacity with a certain fairness constraint. By formulating and simplifying the target problem, we have proposed a new solution to exploiting the portal capacity of WMNs. The centralized part of the solution provides the optimality of the portal capacity. The distributed part maintains this optimality when the system has local minor changes, and it brings robustness to the system as well. In addition, this solution makes the support of multigateway possible, which can significantly improve system capacity.

\section{REFERENCES}

[1] I. Akyildiz, X. Wang, and W. Wang, "Wireless mesh networks: A survey," Comput. Netw., vol. 47, no. 4, pp. 445-487, Mar. 2005.

[2] H. Jiang, P. Wang, W. Zhuang, and X. Shen, "An interference aware distributed resource management scheme for CDMA-based wireless mesh backbone," IEEE Trans. Wireless Commun., vol. 6, no. 12, pp. 4558-4567, Dec. 2007.

[3] P. Kyasanur and N. H. Vaidya, "Capacity of multi-channel wireless networks: Impact of number of channels and interfaces," in Proc. Mobicom, Cologne, Germany, Aug. 2005, pp. 43-57.

[4] A. H. M. Rad and V. W. S. Wong, "Joint channel allocation, interface assignment and MAC design for multi-channel wireless mesh networks," in Proc. Infocom, Anchorage, AK, May 2007, pp. 1469-1477.

[5] M. Alicherry, R. Bhatia, and L. Li, "Joint channel assignment and routing for throughput optimization in multi-radio wireless mesh networks," in Proc. Mobicom, Cologne, Germany, Aug. 2005, pp. 58-72.

[6] M. Kodialam and T. Nandagopal, "Characterizing the capacity region in multi-radio multi-channel wireless mesh networks," in Proc. Mobicom, Cologne, Germany, Aug. 2005, pp. 73-87.

[7] K. N. Ramachandran, E. M. Belding, K. C. Almeroth, and M. M. Buddhikot, "Interference-aware channel assignment in multiradio wireless mesh networks," in Proc. Infocom, Barcelona, Spain, Apr. 2006, pp. 1-12.

[8] A. Raniwala and T. Chiueh, "Architecture and algorithms for an IEEE 802.11-based multi-channel wireless mesh network," in Proc. Infocom, Miami, FL, Mar. 2005, pp. 2223-2234.

[9] K. Xing, X. Cheng, L. Ma, and Q. Liang, "Superimposed code based channel assignment in multi-radio multi-channel wireless mesh networks," in Proc. Mobicom, Montreal, QC, Canada, Sep. 2007, pp. 15-26.

[10] W. Tam and Y. Tseng, "Joint multi-channel link layer and multi-path routing design for wireless mesh networks," in Proc. Infocom, Anchorage, AK, May 2007, pp. 2081-2089.

[11] H. Zhai, Y. Kwon, and Y. Fang, "Performance analysis of IEEE 802.11 MAC protocols in wireless LANs," Wireless Commun. Mobile Comput., vol. 4, no. 8, pp. 917-931, Nov. 2004.

[12] G. Bianchi, "Performance analysis of the IEEE 802.11 distributed coordination function," IEEE J. Sel. Areas Commun., vol. 18, no. 3, pp. 535-547, Mar. 2000.

[13] S. Wu, C. Lin, Y. Tseng, and J. Sheul, "A new multi-channel MAC protocol with on-demand channel assignment for multi-hop mobile ad hoc networks," in Proc. ISPAN, Washington, DC, Dec. 2000, pp. 232-237.

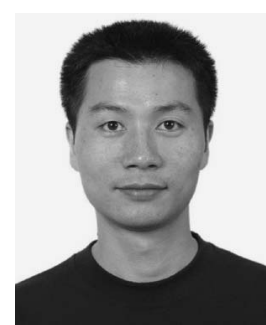

Rongsheng Huang ( $\mathrm{S}^{\prime}$ '07) received the B.S. and M.S. degrees in electrical engineering from Xi'an Jiaotong University, Xi' an, China, in 1996 and 1999, respectively. $\mathrm{He}$ is currently working toward the Ph.D. degree with the Department of Electrical and Computer Engineering, University of Florida, Gainesville.

From 1999 to 2001, he was with Huawei Technologies Co. Ltd. as an R\&D Engineer on GPRS and $3 \mathrm{G}$ projects. From 2002 to 2005 , he was with UTStarcom Research Center, Shenzhen, China, as a Senior Engineer and Team Leader on $3 \mathrm{G}$ projects. His research interests are in the area of media access control, protocol, and architecture for wireless networks.

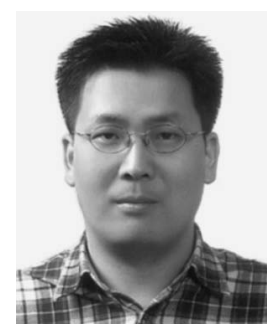

Sunmyeng Kim received the B.S., M.S., and Ph.D. degrees from Ajou University, Korea, in 2000, 2002 , and 2006, respectively, all in information and communication.

He was a Postdoctoral Researcher with the Department of Electrical and Computer Engineering, University of Florida, Gainesville, from May 2006 to February 2008. He then joined the School of Computer and Software Engineering, Kumoh National Institute of Technology, Gumi, Korea, as a fulltime lecturer in March 2008. His research interests include resource management, wireless LANs and PANs, wireless mesh networks, and quality-of-service enhancement.

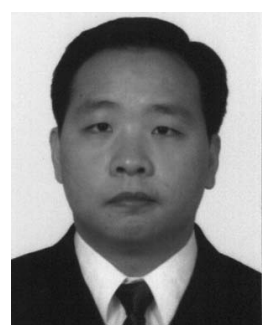

Chi Zhang (S'06) received the B.E. and M.E. degrees in electrical engineering from Huazhong University of Science and Technology, Wuhan, China, in 1999 and 2002, respectively. He is currently working toward the Ph.D. degree with the Department of Electrical and Computer Engineering. University of Florida, Gainesville.

His research interests are network and distributed system security, wireless networking, and mobile computing, with emphasis on mobile ad hoc networks, wireless sensor networks, wireless mesh networks, and heterogeneous wired/wireless networks.

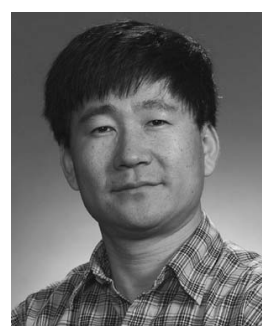

Yuguang Fang (S'92-M'93-SM'99-F'08) received the $\mathrm{Ph} . \mathrm{D}$. degree in systems engineering from Case Western Reserve University, Cleveland, OH, in 1994 and the Ph.D. degree in electrical engineering from Boston University, Boston, MA, in 1997.

He was an Assistant Professor with the Department of Electrical and Computer Engineering, New Jersey Institute of Technology, Newark, from July 1998 to May 2000. He then joined the Department of Electrical and Computer Engineering, University of Florida, Gainesville, in May 2000 as an Assistant Professor, got an early promotion to Associate Professor with tenure in August 2003 and to Full Professor in August 2005. He held a University of Florida Research Foundation Professorship from 2006 to 2009 and is holding a Changjiang Scholar Chair Professorship from 2008 to 2011 with Xidian University, Xi' an, China. He has published more than 250 papers in refereed professional journals and conferences.

Dr. Fang is the Editor-in-Chief of IEEE WIRELESS COMMUNICATIONS and serves/has served on several editorial boards of technical journals, including the IEEE TRANSACTIONS ON Mobile COMPUTING, IEEE TRANSACTIONS ON COMMUNICATIONS, IEEE TRANSACTIONS ON Wireless COMMUNICATIONS, IEEE WIRELESS COMMUNICATIONS, and ACM Wireless Networks. He has also been actively participating in professional conference organizations, such as serving as the Technical Program Vice Chair for the IEEE Conference on Computer Communications (INFOCOM) 2005, the Technical Program Symposium Cochair for the IEEE Global Telecommunications Conference 2004, and a Technical Program Committee Member for the IEEE INFOCOM (1998, 2000, and 2003-2009), ACM International Symposium on Mobile Ad Hoc Networking and Computing (2008-2009), and ACM International Conference on Mobile Computing and Networking (2001). He was the recipient of the National Science Foundation Faculty Early Career Award in 2001 and the Office of Naval Research Young Investigator Award in 2002. 